03

\title{
Измерение существенно нестационарных тепловых потоков градиентным датчиком на основе висмута
}

\author{
( Ю.В. Добров, ${ }^{1}$ В.А. Лашков, ${ }^{1}$ И.Ч. Машек,, А.В. Митяков, ${ }^{2}$ В.Ю. Митяков, ${ }^{2}$ \\ C.3. Сапожников, ${ }^{2}$ P.С. Хоронжук ${ }^{1}$ \\ ${ }^{1}$ Санкт-Петербургский государственный университет, \\ 199034 Санкт-Петербург, Россия \\ ${ }^{2}$ Санкт-Петербургский политехнический университет Петра Великого, \\ 195251 Санкт-Петербург, Россия \\ e-mail: youdobrov@gmail.com
}

Поступило в Редакцию 22 июня 2020 г.

В окончательной редакции 1 сентября 2020 г.

Принято к публикации 1 сентября 2020 г.

Выполнена калибровка градиентного датчика теплового потока, изготовленного из монокристалла висмута. Найдено значение вольт-ваттной чувствительности датчика и представлен метод обработки данных, основанный на одномерном уравнении теплопроводности для тонкой пластины. Полученный метод был апробирован на экспериментальных данных, полученных при инициации лазерного разряда в спокойной атмосфере и в сверхзвуковом потоке газа.

Ключевые слова: нестационарные тепловые потоки, градиентный датчик, нестационарная теплометрия.

DOI: $10.21883 /$ JTF.2021.02.50357.209-20

\section{Введение}

Изучение нагрева летательного аппарата является одним из основных направлений исследований экспериментальной аэродинамики. В связи с повышением скоростей полета проблема теплопередачи становится значительной и актуальной. В настоящее время особенно интересна разработка способов и устройств для измерения быстро меняющихся во времени тепловых потоков.

При измерении тепловых потоков в высокотемпературных газовых потоках обычно применяются калориметрические датчики [1], которые используются по типу вспомогательной стенки. Элементарная теория такого датчика теплового потока основана на стационарном решении краевой задачи теплопроводности для неограниченной пластины. В соответствии с такой постановкой задачи тепловой поток в любом сечении этой пластины постоянен [2]. Основной недостаток калориметрического метода при измерении высокочастотных тепловых потоков - большая неопределенность результатов измерений из-за погрешности измерения температуры чувствительного элемента [3].

В последнее время получил распространение новый тип датчиков на основе поперечного эффекта Зеебека $[4,5]$, который определяется генерацией в анизотропной среде ЭДС в направлении, перпендикулярном градиенту температур [6,7]. Эти датчики привлекают высокой чувствительностью и быстродействием [8]. Следует выделить появление новой технологии создания градиентных датчиков теплового потока (ГДТП), в которых использованы анизотропные монокристаллы висмута высокой чистоты $[9,10]$. ГДТП могут работать в широ- ком диапазоне тепловых потоков, температур, давлений и обладают высокими частотными характеристиками (до $20 \mathrm{kHz}$ ). Датчики обладают малым сопротивлением $(1-10 \Omega)$, что важно для обеспечения помехозащищенности измерительной цепи при измерениях в сильных импульсных электромагнитных полях. Датчик ГДТП может надежно использоваться для прямого определения теплового потока, если применяется в измерениях с характерным временем более $20 \mathrm{~ms}$ [11]. Важно отметить, что постоянная времени датчика не зависит от его толщины, а определяется только конструкцией и свойствами материалов, и для ГДТП на основе висмута составляет $50 \mu \mathrm{s}$. Предельно достижимое значение $10 \mu \mathrm{s}[12,13]$.

Для исследования нестационарных воздействий, приложенных к датчику теплового потока, необходимо установить взаимосвязь между величинами входного теплового потока на датчик и его реакцией на это воздействие - выходным электрическим сигналом, который регистрируется измерительной системой. Обсуждаются разнообразные алгоритмы обработки сигнала датчика, основанные на разных моделях теплопроводности $[3,11]$ датчика и особенностях генерации термоЭДС [14]. В [11] сравниваются результаты обработки сигнала ГДТП с результатами прямого измерения датчиком ALTP (FORTECH HTS GmbH, Германия), используемым для прямого измерения теплового потока в процессах с характерным временем более $10 \mu \mathrm{s}$. Утверждается, что требуется детализация теплообмена датчика с элементами конструкции и, возможно, учет двумерных эффектов. В [14] вводится понятие эффективной толщины зоны генерации. Из проведенного в 
работе опыта видно, что в среднем толщина этой зоны, постепенно нарастая, составляет примерно половину толщины термоэлемента. Основное изменение толщины зоны генерации происходит в начале процесса, когда резко возрастает тепловой поток. Когда же тепловой поток снижается до малого уровня и мало меняется, толщина зоны генерации продолжает расти. В качестве тепловой модели использовано одномерное нестационарное уравнение теплопроводности для полупространства, и по найденному значению температуры рабочей поверхности датчика рассчитывался тепловой поток. Однако существуют экспериментальные исследования, которые показывают, что постоянная времени датчика не зависит от его толщины [12].

В настоящей работе приведен квазистатический метод калибровки ГДТП, предложен алгоритм обработки сигнала датчика и показано применение ГДТП на основе висмута для исследования тепловых потоков на аэродинамическом теле при локальном нагреве сверхзвукового набегающего воздуха. Лазерный или СВЧ разряды используются в аэродинамическом эксперименте для управления обтеканием тела [15]. Лазерный пробой организуется перед моделью на оси, нагретая область газа набегает на тело. Время взаимодействия нагретой области газа с моделью составляет порядка $100 \mu \mathrm{s}$. Характерное время порядка $1-10 \mu \mathrm{s}$. Алгоритм обработки основан на применении метода нестационарной теплопроводности с граничными условиями второго рода. Такой подход позволяет определить тепловой поток на лицевой поверхности датчика.

\section{1. Квазистатическая калибровка датчика теплового потока}

В настоящей работе использовался ГДТП, изготовленный в Санкт-Петербургском государственном политехническом университете. Основой для датчика являются анизотропные термоэлементы из монокристалла висмута, имеющие форму прямоугольного параллелепипеда с толщиной и шириной равными $0.2 \mathrm{~mm}$, длиной $l=2 \mathrm{~mm}$. Десять таких термоэлементов образуют пластину с площадью лицевой поверхности $2 \times 2 \mathrm{~mm}$ и толщиной $h=0.2 \mathrm{~mm}$. Термоэлементы последовательно соединены в электрическую цепь. Датчик оснащен дифференциальным усилителем, собранным по двухкаскадной схеме, с коэффициентом усиления равным 175 и характерным временем 25-30 ns.

Чтобы найти вольт-ваттную чувствительность измерительной системы, использовался квазистационарный лучистый тепловой поток. В качестве источника излучения применялась галогеновая лампа мощностью $500 \mathrm{~W}$. Оптической системой создавался равномерный тепловой поток на поверхности ГДТП. Лучистый поток, поступающий от источника на датчик, формировался при помощи модулятора. С помощью фотодиода ФД-24К

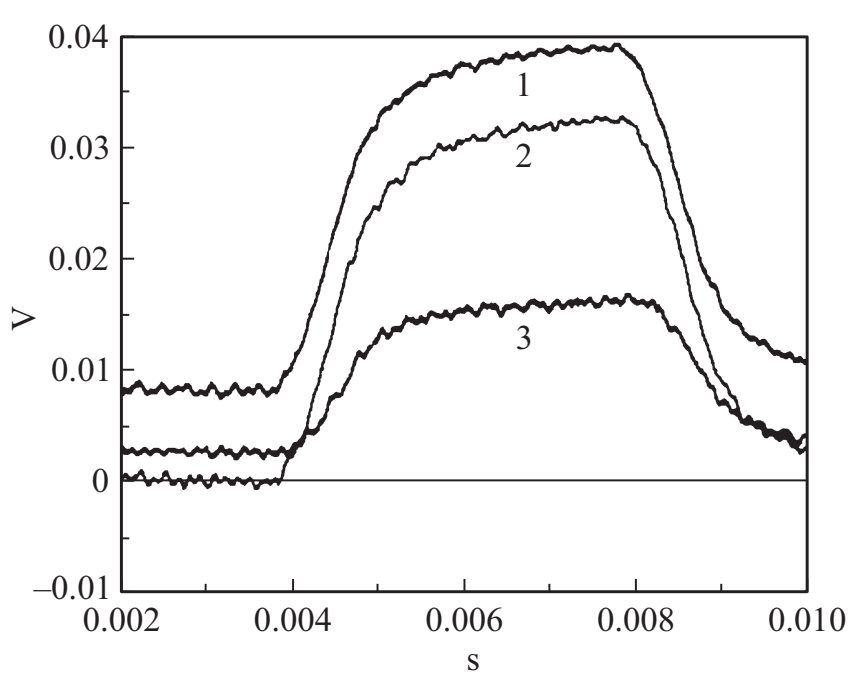

Рис. 1. Данные с ГДТП в экспериментах № 1-3.

Экспериментально измеренные значения потока мощности и сосчитанная вольт-ваттная чувствительность в серии экспериментов

\begin{tabular}{c|c|c}
\hline № эксперимента & $\begin{array}{c}\text { Поток мощности } \\
\text { на ГДТП, } \mathrm{mW}\end{array}$ & $\begin{array}{c}\text { Чувствительность ИС, } \\
\mathrm{mV} / \mathrm{W}\end{array}$ \\
\hline 1 (без фильтров) & 40 & 750 \\
2 (КС-19) & 30.3 & 726 \\
3 (ИКС-3) & 18.4 & 652
\end{tabular}

было установлено, что сигнал модулируемого потока мощности имеет форму равнобедренной трапеции.

Для ослабления потока мощности и выяснения возможного влияния фотоэффекта было проведено несколько экспериментов: сначала лучистый поток подавался непосредственно от источника (эксперимент № 1), а затем через инфракрасные фильтры КС-19 и ИКС-3 (эксперименты № 2 и № 3). Для исключения возможных переотражений фильтры наклонялись по углом $20^{\circ}$ к оптической оси. Мощность излучения находилась при помощи измерителя средней мощности ИМО-2. Показания с ГДТП записывались с частотой $0.5 \mathrm{MHz}$, полученные экспериментальные данные представлены на рис. 1.

Очевидно, что графики близки по форме к криволинейной трапеции. Можно отметить, что при поступлении на ГДТП в течение $5 \mathrm{~ms}$ стационарного теплового потока напряжение не остается на одном уровне и продолжает понемногу возрастать, а также затянутые во времени передний и задний фронты.

Зная поступающую на датчик мощность излучения и значение максимального отклонения напряжения, можно было найти чувствительность измерительной системы (ГДТП с усилителем). Экспериментально замеренный поток мощности на ГДТП и чувствительность измерительной системы представлены в таблице. 

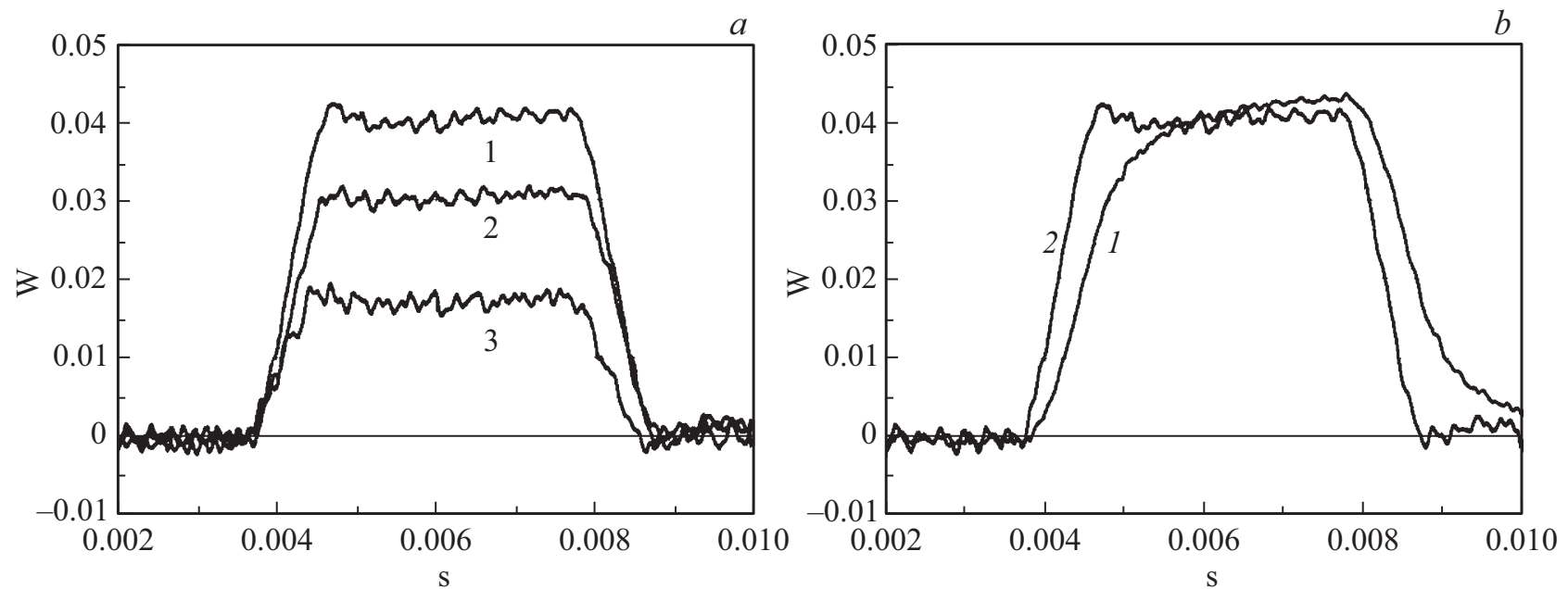

Рис. 2. $a-$ расчетный тепловой поток в экспериментах № $1-3 ; b-$ сравнение экспериментального (1) с поправкой на чувствительность и расчетного (2) тепловых потоков из эксперимента № 1.

Таким образом, было установлено, что среднее значение вольт-ваттной чувствительности измерительной системы составляет $709 \mathrm{mV} / \mathrm{W}$. Погрешность измерения потока мощности на датчик составляет порядка 7\%. Хорошая корреляция чувствительности в трех спектральных диапазонах подтверждает отсутствие значительного влияния фотоэлектрического эффекта на сигнал приемника и высокую степень его серости.

\section{2. Построение математической модели датчика и ее апробация}

В предположении, что вектор градиента температуры направлен перпендикулярно поверхности датчика, будем использовать формулу Томсона [14] для связи разности температур тыльной и лицевой поверхностей с напряжением, получаемым на ГДТП

$$
U=\alpha_{x y}\left(T_{h}-T_{0}\right) \frac{l k}{h}
$$

где $U$ - возникающее на датчике напряжение, $\alpha_{x y}-$ компонента тензора термоЭДС висмута, $T_{h}$ - температура лицевой поверхности датчика, $T_{0}$ - температура тыльной поверхности датчика, $k-$ количество термоэлементов в датчике. То есть разница температур пропорциональна возникающему напряжению.

На рис. 2, $b$ представлено сравнение экспериментальных данных с поправкой на вольт-ваттную чувствительность, полученных из эксперимента № 1, с расчетным тепловым потоком. Можно отметить хорошее совпадение сигналов по амплитуде. С учетом работы модулятора тепловой поток соответствует форме сигнала мощности лучистой энергии, подаваемой на ГДТП. Использованная методика позволяет хорошо описать временные особенности теплового потока, регистрируемого ГДТП.
Учитывая достаточно маленькую толщину датчика (отношение толщины датчика к его ширине составляет 1/10), опишем процесс теплообмена ГДТП с внешней средой при помощи уравнения теплопроводности для бесконечно тонкой пластины с граничными условиями второго рода. Датчик установлен на основании с низким коэффициентом теплопроводности (полиэтилен), поэтому будем считать, что тепловой поток на тыльной стороне отсутствует, и изначально температура имеет равномерное распределение. Запишем решение уравнения нестационарного теплообмена для пластины в форме, предложенной в [16]:

$$
\begin{aligned}
& T(x, \tau)-T_{s}=\frac{\alpha}{\lambda h} \int_{0}^{\tau} q(\vartheta) d \vartheta+\frac{2 \alpha}{\lambda h} \sum_{n=1}^{\infty}(-1)^{n} \\
& \times \cos \left(\mu_{n} \frac{x}{h}\right) \int_{0}^{\tau} q(\vartheta) \exp \left[-\frac{\alpha_{n}^{2}}{h^{2}}((t-\vartheta)] d \vartheta,\right.
\end{aligned}
$$

где $T(x, \tau)$ - температура пластины в зависимости от толщины и времени, $T_{s}$ - начальное распределение температуры в пластине, $\alpha-$ температуропроводность, $\lambda$ - теплопроводность висмута, $q(t)$ - тепловой поток к лицевой поверхности пластины в зависимости от времени, коэффициент $\mu_{n}=\pi n$. Запишем выражение (2) для $x=h, x=0$, вычтем одно из другого и получим

$$
\begin{aligned}
T_{h}(\tau) & -T_{0}(\tau)=\frac{2 \alpha}{\lambda h} \sum_{n=1}^{\infty}(-1)^{n}\left(\cos \left(\mu_{n}\right)-1\right) \\
& \times \int_{0}^{\tau} q(\vartheta) \exp \left[-\frac{\alpha \mu_{n}^{2}}{h^{2}}(t-\vartheta)\right] d \vartheta .
\end{aligned}
$$

Зная из эксперимента значение напряжения на датчике в каждый момент времени, мы находим разницу 


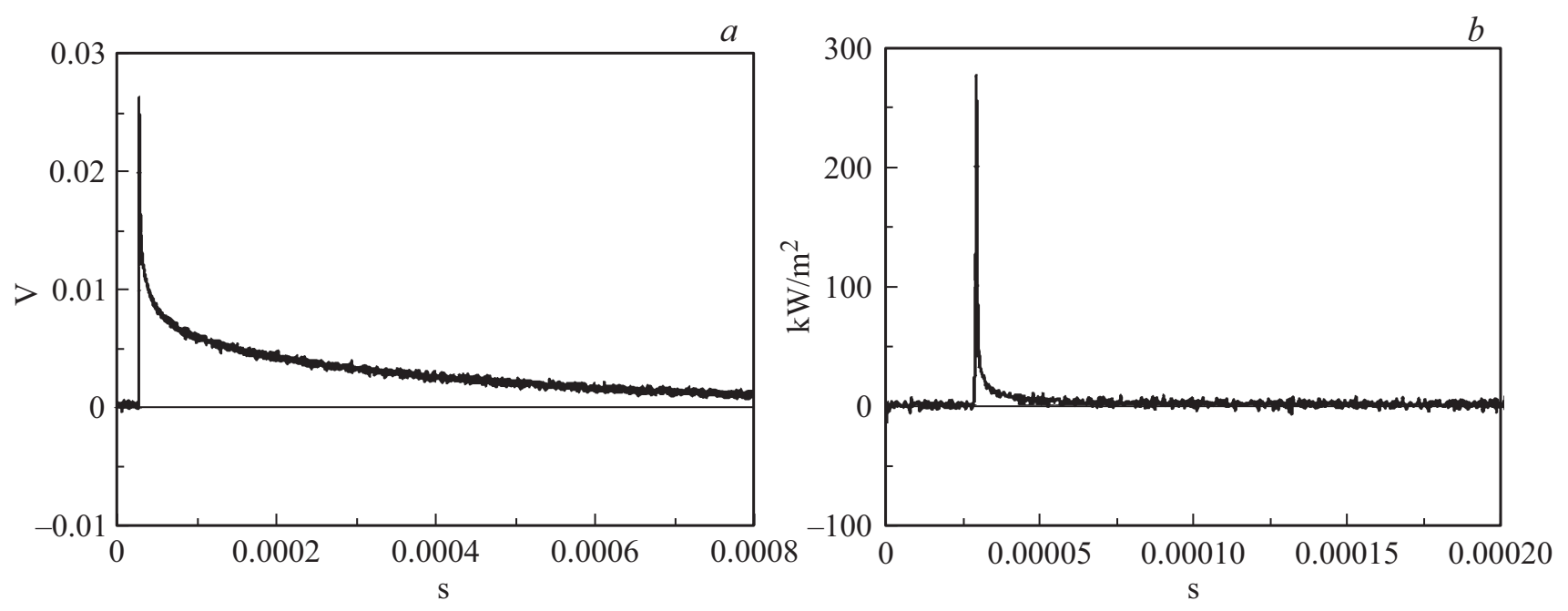

Pис. 3. $a-$ данные с ГДТП при воздействии лазерной искрой; $b-$ расчетная плотность теплового потока в эксперименте с лазерной искрой.

между температурами $T_{h}$ и $T_{0}$ из уравнения (1). Затем. численно решая обратную интегральную задачу (3), можем найти значение теплового потока для каждого момента времени.

Восстановив этим методом значения теплового потока из имеющихся экспериментальных данных (рис. 1), получим, что график теплового потока в зависимости от времени имеет форму прямоугольной трапеции (рис. 2,a).

\section{3. Исследование теплового потока на затупленном цилиндре в сверхзвуковом потоке}

Исследования проводились на сверхзвуковой аэродинамической трубе, имеющей рабочую камеру Эйфеля, в которой с помощью профилированного сопла создавался рабочий поток с числом Маха, равным 1.5. Давление в камере поддерживалось на уровне 155 Torr с помощью сверхзвукового эжектора. Диаметр рабочего потока $60 \mathrm{~mm}$. Температура торможения потока $288 \mathrm{~K}$. Аэродинамическая модель представляет собой цилиндр с диаметром $12 \mathrm{~mm}$ и высотой $20 \mathrm{~mm}$, изготовленный из фторопласта. Датчик устанавливался на аэродинамической модели в центральной части лобовой поверхности. Сверхзвуковой рабочий поток подвергался модификации с помощью импульсного лазера EverGreen-145Nd:YAG. Длина волны $-532 \mathrm{~nm}$, энергия в импульсе - $145 \mathrm{~mJ}$. На оси потока на расстоянии $40 \mathrm{~mm}$ перед моделью с использованием лазера создавалась искра.

Работы по исследованию влияния плазменного образования в сверхзвуковом потоке перед телом с целью изменения его аэродинамических характеристик проводятся у нас в стране и за рубежом многими авторами, например $[15,17,18]$. Плазмоид создается с помощью раз- ного вида разрядов (электрического, лазерного, СВЧ). Установлено, что основным фактором, влияющим на изменение аэродинамических характеристик тела, является локальный нагрев газа. Основные работы посвящены изучению влияния локального нагрева на изменение силовых и тепловых нагрузок на тело. И если изменение силовых нагрузок на теле в этих условиях достаточно хорошо исследовано, то поведение тепловых потоков на поверхности тела еще требует изучения.

Исследования теплового потока на модели начинались с опытов, которые проводились в спокойной атмосфере в рабочей камере и при давлении 155 Torr. Лазерная искра создавалась на расстоянии $80 \mathrm{~mm}$ от модели. Показания снимались с частотой $2.5 \mathrm{MHz}$. На рис. 3, $a$ показана реакция измерительной системы на тепловой поток, идущий от лазерной искры без рабочего потока.

Измерительная система довольно быстро реагирует на тепловой поток от лазерного разряда, сигнал резко возрастает с появлением лазерной искры. Рост сигнала происходит за 15-20 ns. Можно отметить, что начальная фаза сигнала (рост и последующий спад примерно до половины амплитуды) протекает существенно быстрее, чем его „хвост“, на котором наблюдается длительное монотонное уменьшение сигнала. Если обработать этот сигнал предложенным способом, то мы получим следующую картину (рис. $3, b$ ).

Обработка экспериментальных данных избавляет от длинного „хвоста“ и дает качественно более близкий к реальному график теплового потока от искры лазера. В работе [19] показано, что интенсивное выделение тепла происходит в первые десятки наносекунд после оптического пробоя, а затем следует практически мгновенный спад температуры и энергии излучения.

Очевидно, что проведение измерения теплового потока от искры лазерного излучения с частотой $2.5 \mathrm{MHz}$ (время между измерениями $400 \mathrm{~ns}$ ) очень грубо опи- 

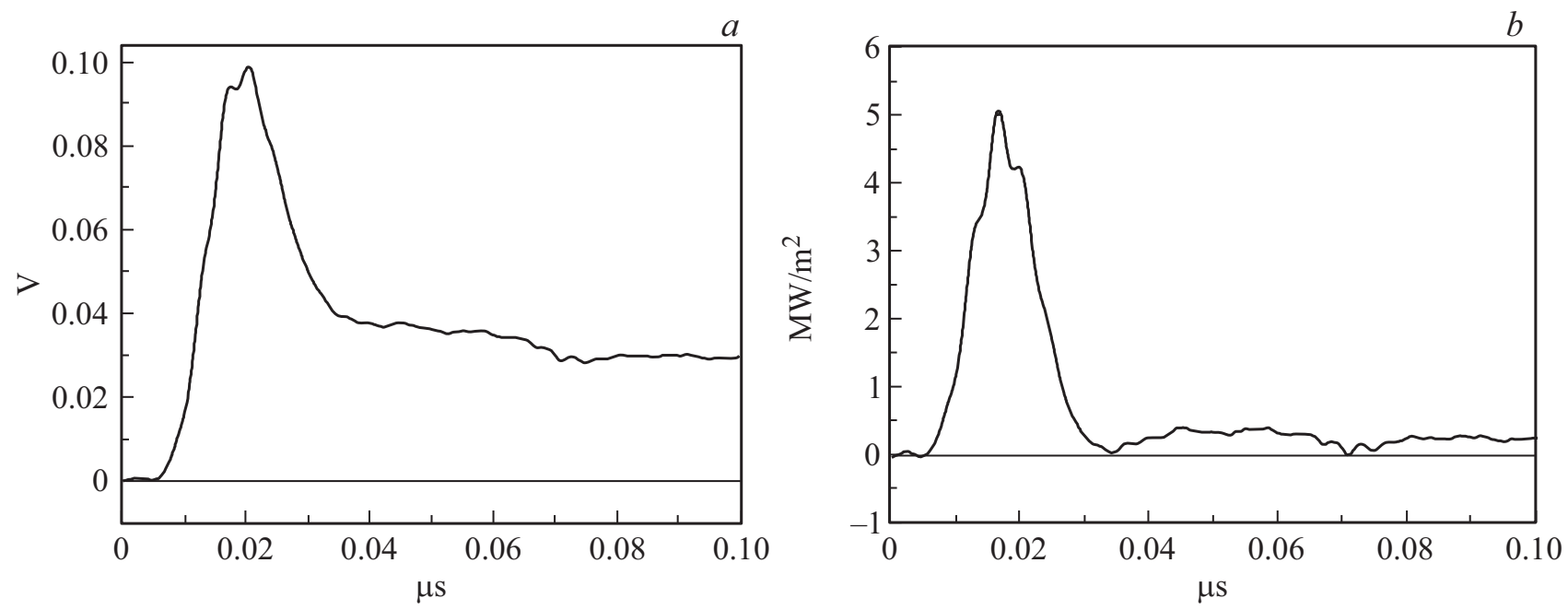

Рис. 4. $a$ - данные с ГДтП при увеличении частоты регистрации до $25 \mathrm{GHz} ; b-$ расчетная плотность теплового потока.
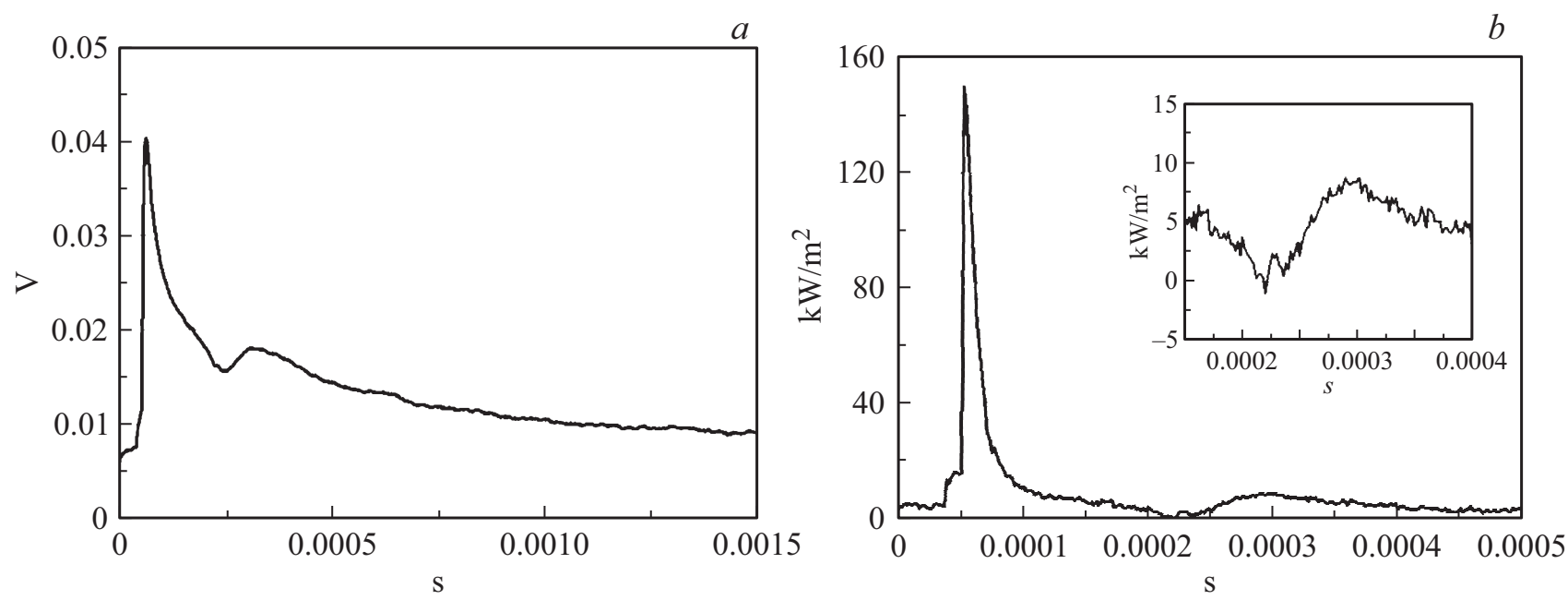

Pис. 5. $a-$ реакция ГДТП на разряд в сверхзвуковом потоке газа; $b-$ расчетное изменение плотности теплового потока.

сывает начальную активную фазу разряда, который продолжается несколько десятков наносекунд. Поэтому были проведены измерения с повышенной частотой снятия показаний $-25 \mathrm{GHz}$ (рис. 4,a). Такой подход позволил более подробно зафиксировать сигнал с ГДТП. Можно отметить более высокую амплитуду изменения напряжения, зафиксированную ГДТП.

На рис. 4, $b$ показана плотность теплового потока от лазерной искры, полученная с помощью обработки сигнала ГДТП с использованием предложенной методики. Плотность теплового потока достигает величины $5 \mathrm{MW} / \mathrm{m}^{2}$. Предложенная методика позволяет с хорошей точностью восстановить тепловые потоки, действующие на малых временах (порядка нескольких наносекунд).

Затем исследовалась реакция ГДТП на разряд лазера в сверхзвуковом потоке (рис. 5,a). В этом случае, помимо лучистого теплового потока от искры, ГДТП будет фиксировать конвективный теплообмен на модели от следа разряда.

В этом эксперименте использовался усилитель сигнала ГДТП с частотой пропускания $20 \mathrm{kHz}$. Это отражается в показаниях датчика (рис. $3, a$ и рис. $5, a$ ). На рис. $3, a$ пик сигнала датчика более острый, чем на рис. 5, a. Таким образом, ГДТП на основе висмута с использованием предложенной методики обработки сигнала позволяет исследовать высокоскоростные тепловые потоки, а усилитель сигнала должен обладать быстродействием, соответствующим изучаемому процессу.

На рис. 5, $b$ представлен результат обработки сигнала ГДТП с помощью описанной методики. На модели в критической точке в стационарных условиях обтекания наблюдается постоянный тепловой поток. Величина плотности теплового потока составляет около $5 \mathrm{~kW} / \mathrm{m}^{2}$. Примерно на $200 \mu$ s нагретая область газа после лазерной искры начинает взаимодействовать с ударным слоем 
на модели. Структура течения около модели изменяется, изменяется и тепловой поток на лобовой поверхности. При этом сначала тепловой поток падает практически до нуля, затем увеличивается и более чем в два раза превышает обычный тепловой поток. Затем обтекание модели восстанавливается, и тепловой поток уменьшается до стационарного уровня. Весь процесс изменения теплового потока занимает во времени около $200 \mu \mathrm{s}$. Таким образом, в результате взаимодействия следа лазерного разряда с ударным слоем перед телом характер течения у поверхности модели меняется настолько значительно, что за счет изменения конвективного теплообмена происходит кратковременное падение и затем увеличение плотности теплового потока.

\section{Заключение}

Проведена экспериментальная калибровка измерительной системы (ГДТП усилитель) и найдена ее вольтваттная чувствительность. Предложена модель работы ГДТП, необходимая для обработки экспериментальных данных, полученных с помощью этого датчика. Результаты расчетов теплового потока по предложенной модели хорошо коррелируют с данными экспериментальных исследований ГДТП.

Полученный метод был апробирован при исследовании теплового потока от лазерной искры, инициируемой в спокойной атмосфере и в сверхзвуковом потоке газа. В случае лазерного разряда в спокойной атмосфере датчик регистрирует только лучистый тепловой поток от распадающейся плазмы. В случае сверхзвукового обтекания цилиндра тепловой поток изменяется как за счет поступающего от искры излучения, так и при взаимодействии нагретой лазерным разрядом области газа с ударным слоем на теле. В последнем случае существенно перестраивается течение и меняется температура газа у лобовой поверхности экспериментальной модели, что приводит к значительному изменению конвективного теплового потока.

Предложенная методика обработки сигнала ГДТП хорошо показала себя при исследовании как стационарных, так и быстропротекающих тепловых процессов. Построенная измерительная система на базе ГДТП и усилителя обладает высокой чувствительностью и хорошим временным разрешением порядка нескольких наносекунд, что позволяет проводить исследования широкого спектра физических явлений.

\section{Финансирование работы}

Работа выполнена при поддержке РФФИ (гранты № 19-31-90071 и 18-08-00707). При расчетах использовались компьютерные ресурсы, предоставленные РЦ „Вычислительный центр СПбГУ“.

\section{Конфликт интересов}

Авторы заявляют, что у них нет конфликта интересов.

\section{Список литературы}

[1] Н.Ф. Краснов, В.Н. Кошевой, А.Н. Данилов. Прикладная аэродинамика. Под ред. Краснова Н.Ф. Уиеб. пособие для втузов (Высшая школа, М., 1974)

[2] А.М. Харитонов. Техника и методы аэрофизического эксперимента. Ч. 2. Методы и средства аэрофизических измерений: учебник (Изд-во НГТУ, Новосибирск, 2007)

[3] Н.В. Пилипенко. Основы проектирования комбинированных приемников теплового потока. Уиебное пособие (Университет ИТМО, СПб, 2016)

[4] T. Roediger, H. Knauss, D. Bountin, B. Smorodsky, A. Maslov, J. Srulijes, J. Spacecraft Rockets, 46 (2), 255 (2009). DOI: $10.2514 / 1.32011$

[5] С.3. Сапожников, В.Ю. Митяков, А.В. Митяков. ЖТФ, 74 (7), 114 (2004). [S.Z. Sapozhnikov, V.Y. Mitiakov, A.V. Mitiakov. Tech. Phys., 49 (7), 920 (2004). DOI: $10.1134 / 1.1778869]$

[6] А.А. Снарский, А.М. Пальти, А.А. Ащеулов. ФТП, 31 (11), 1281 (1997).

[7] А.Г. Самойлович. Термоэлектрические и термомагнитные методы превращения энергии: Конспект лекций (Изд-во ЛКИ, М., 2007)

[8] С.В. Бобашев, Ю.П. Головачев, Н.П. Менде, П.А. Попов, Б.И. Резников, В.А. Сахаров, А.А. Шмидт, А.С. Чернышев, С.З. Сапожников, В.Ю. Митяков, А.В. Митяков. ЖТФ, $\mathbf{7 8}(12), 103$ (2008).

[9] С.З. Сапожников, В.Ю. Митяков, А.В. Митяков. Градиентные датчики теплового потока (Изд-во СПбГПУ, СПб, 2003)

[10] Т.Г. Грищенко, Л.В. Декуша, Л.И. Воробьев. Теплометрия: теория, метрология, практика. Монография в трех книгах. Кн. 1: Методы и средства измерения теплового потока (Институт технической теплофизики НАН Украины, Киев, 2017)

[11] С.В. Бобашев, Н.П. Менде, П.А. Попов, Б.И. Резников, В.А. Сахаров, С.3. Сапожников, В.Ю. Митяков, А.В. Митяков, Д.А. Бунтин, А.А. Маслов, Х. Кнаусс, Т. Редигер. Письма в ЖТФ, 35 (5), 36 (2009).

[12] А.В. Митяков. Градиентные датчики теплового потока в нестационарной теплометрии (Автореф. канд. дисс., СПбПУ, СПб., 2000)

[13] С.3. Сапожников, В.Ю. Митяков, А.В. Митяков. ТВТ, 42 (4), 626 (2004).

[14] П.А. Попов, Б.И. Резников, В.А. Сахаров, А.С. Штейнберг. Письма в ЖТФ, 37 (1), 26 (2011).

[15] V.A. Lashkov, I.Ch. Mashek, V.I. Ivanov, Yu.F. Kolesnichenko, M.I. Rivkin. Gas-dynamic peculiarities of microwave discharge interaction with shock wave near the body. AIAA 2008-1410, 46th AIAA Aerospace Sciences Meeting and Exhibit, Reno, NV, 7-11 January, DOI: 10.2514/6.2008-1410 (2008).

[16] А.В. Лыков. Теория теплопроводности (Высшая школа, M., 1967) 
[17] D. Knight. J. Aerospace Lab, 10 (2), (2015). DOI: 10.12762/2015.AL10-02

[18] P. Bletzinger, B.N. Ganguly, D. VanWie, A. Garscadden. J. Phys. D: Appl. Phys., 38 (4), R33 (2005). DOI: $10.1088 / 0022-3727 / 38 / 4 / \mathrm{R} 01$

[19] N. Glumac, G. Elliott, M. Boguszko. AIAA J., 43 (9), 1984 (2005). DOI: $10.2514 / 1.14886$ 